\title{
Intraoperative Prevention of Stenosis for Laparoscopic Sleeve Gastrectomy
}

\author{
Ramon Vilallonga, MD, PhD, Jacques Himpens, MD
}

Division of Bariatric Surgery, AZ St. Blasius, Dendermonde, Belgium (all authors).

\begin{abstract}
Introduction: Many different medical and surgical modalities have been described for the treatment of late strictures after laparoscopic sleeve gastrectomy (LSG), including observation, endoscopic dilatations, seromyotomy (SM), and wedge resection of the sleeve stomach including the stricture. Strictures after LSG are preventable with the appropriate surgical technique of the LSG: when stenosis is apparent perioperatively, SM or stricturoplasty (SP) of the stenosis may be performed at that time to prevent the development of a stricture.
\end{abstract}

Case and Technique Description: We present two cases. The first is a 30-year-old morbidly obese woman (body mass index $40 \mathrm{~kg} / \mathrm{m}^{2}$ ) who underwent an LSG at our institution. Stapling was performed over a 34-Fr bougie. After complete transection, a stenosis of the sleeve was observed in the upper part of the stomach. An SP, including a full-thickness longitudinal incision, was performed over the stenotic area and then closed transversely with a full-thickness running suture of resorbable monofilament material $2 / 0$.

The second case is of a 29-year-old woman (body mass index $36 \mathrm{~kg} / \mathrm{m}^{2}$ ) who had a revisional surgery with an adjustable gastric band removal and an LSG performed in one stage at the end of the procedure. A stenosis was detected at the level of the incisura angularis, hence an SM was performed.

In both patients, an omental patch was added over the SP and SM. A drain was left in the abdominal cavity along the SG and remained until discharge from the hospital. A nasogastric tube was placed per routine for 24 hours. The postoperative courses were uneventful for both patients, and they were discharged at postoperative days 3 and 4 , respectively.

Conclusion: An intraoperative approach for iatrogenic stenosis in the primary LSG may include SM and SP. Both techniques are challenging and should avoid chronic and long-term complications related to stenosis during primary LSG.

Key Words: Sleeve gastrectomy, Stricture, Stenosis, Stricturoplasty, Complications management.

Citation Vilallonga R, Himpens J. Intraoperative prevention of stenosis for laparoscopic sleeve gastrectomy. CRSLS e2013.00186. DOI: 10.4293/CRSLS.2013.00186.

Copyright (c) 2015 by SLS, Society of Laparoendoscopic Surgeons. This is an open-access article distributed under the terms of the Creative Commons Attribution-Noncommercial-ShareAlike 3.0 Unported license, which permits unrestricted noncommercial use, distribution, and reproduction in any medium, provided the original author and source are credited.

Dr. Himpens is a consultant for Ethicon Endosurgery and organizes workshops for Gore.

Address correspondence to: Ramon Vilallonga, Division of Bariatric Surgery, AZ St. Blasius, Kroonveldlaan 52, 9200 Dendermonde, Belgium. Telephone: (0034) 626-425-858, Fax: (0032) 052-496-346, E-mail: vilallongapuy@hotmail.com

\section{INTRODUCTION}

Laparoscopic sleeve gastrectomy (LSG) has become an accepted surgical treatment option to manage morbid obesity.1,2 Despite LSG's apparent "simplicity," serious early complications such as bleeding, leaks, and leak complications such as strictures may occur. ${ }^{3}$ Treatment modes of strictures include observation, endoscopic dilation, stricturoplasty (SP), seromyotomy (SM), and wedge resection of the stomach including the stricture, or conversion to laparoscopic Roux-en-Y gastric bypass. ${ }^{3}$ Iatrogenic strictures or stenosis may originate from intraoperative mishaps such as staple misfiring or from scarring after a postoperative complication such as bleeding or a leak. In the intraoperative setting, stricture prevention must be implemented. We present 2 cases in managing inadvertent intraoperative stenosis of the sleeve. 
Intraoperative Prevention of Stenosis for Laparoscopic Sleeve Gastrectomy, Vilallonga R et al.

\section{Case 1}

A 30-year-old morbidly obese woman was admitted to our department to undergo an LSG. Her weight was $100 \mathrm{~kg}$ and her height $158 \mathrm{~cm}$ (body mass index $40 \mathrm{~kg} / \mathrm{m}^{2}$ ). She had no comorbidities.

According to our standard technique, the procedure was performed laparoscopically with 5 trocars. The greater curvature vessels of the stomach were divided at the level of the angulus to reach the lesser sac. The pylorus was identified, and devascularization was continued caudad to $2 \mathrm{~cm}$ proximal to the pylorus. At this stage of the procedure, a stapler (ECHELON 60 ENDOPATH stapler, endoscopic linear cutter [flexible], Ethicon-Endo-Surgery, Cincinnati, Ohio) loaded with black cartridges was used to divide the stomach from the lowest tip of the dissection directed cephalad to the level of the esophagogastric junction.

Stapling was performed over a 34-Fr bougie. After complete transection, the remnant stomach was further devascularized at the greater curve. After removal of the remnant stomach, the sleeve was inspected. In this particular case, a stenosis of the sleeve was observed in the upper part of the stomach. The decision was made to treat the stenosis with an SP (Figure 1A). A full-thickness longitudinal incision was performed over the stenotic area (Figure 1B-D). The incision was closed transversely with a full-thickness running suture of resorbable monofilament material 2/0 to complete the SP (Figure $\mathbf{2 A - C}$ ). The closure of the SP is always performed in a single layer. Air insufflation of the stomach was done at

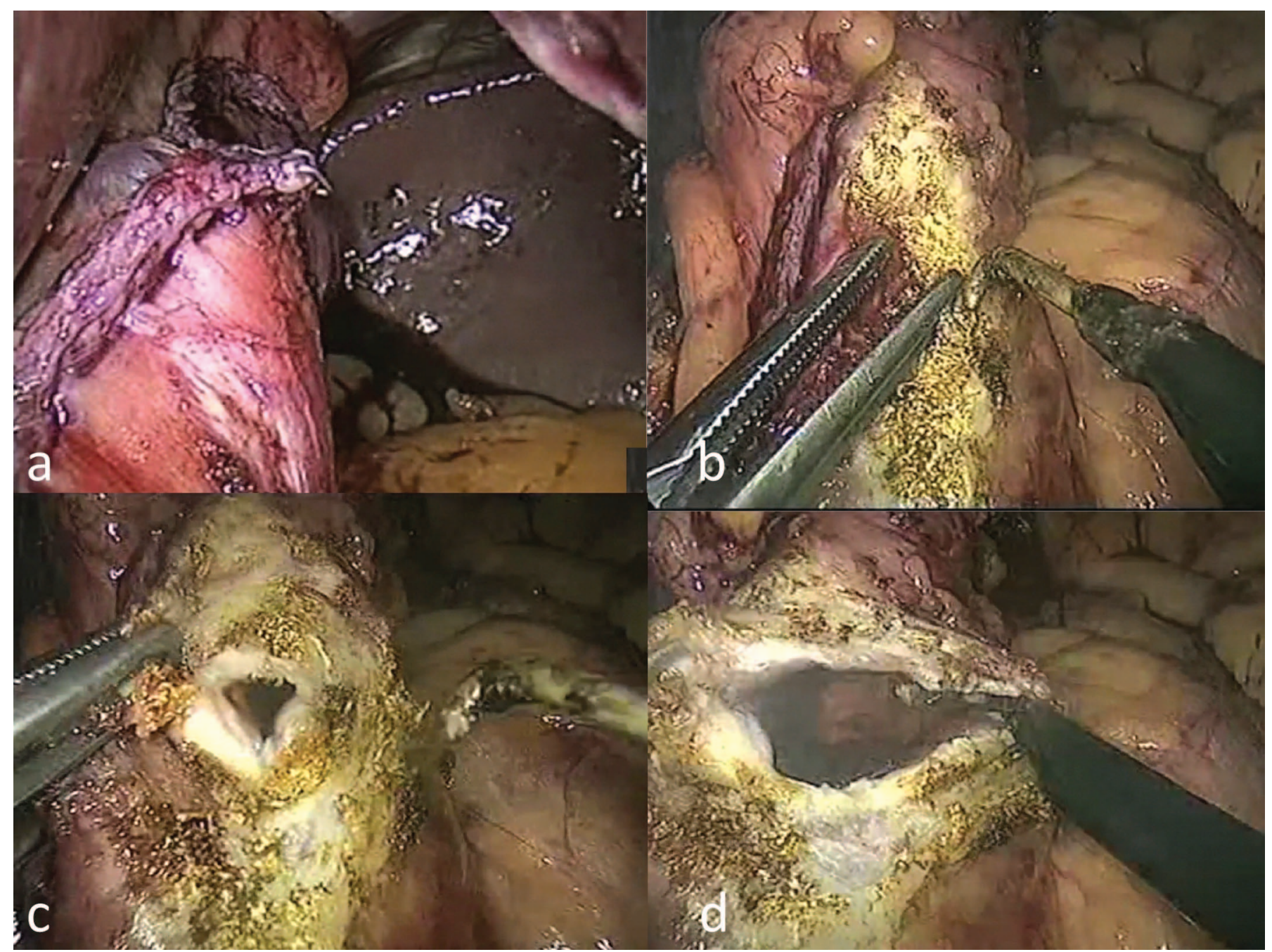

Figure 1. A, Stenotic area of the gastric sleeve. B-D, Full-thickness longitudinal incision performed over the stenotic area. 


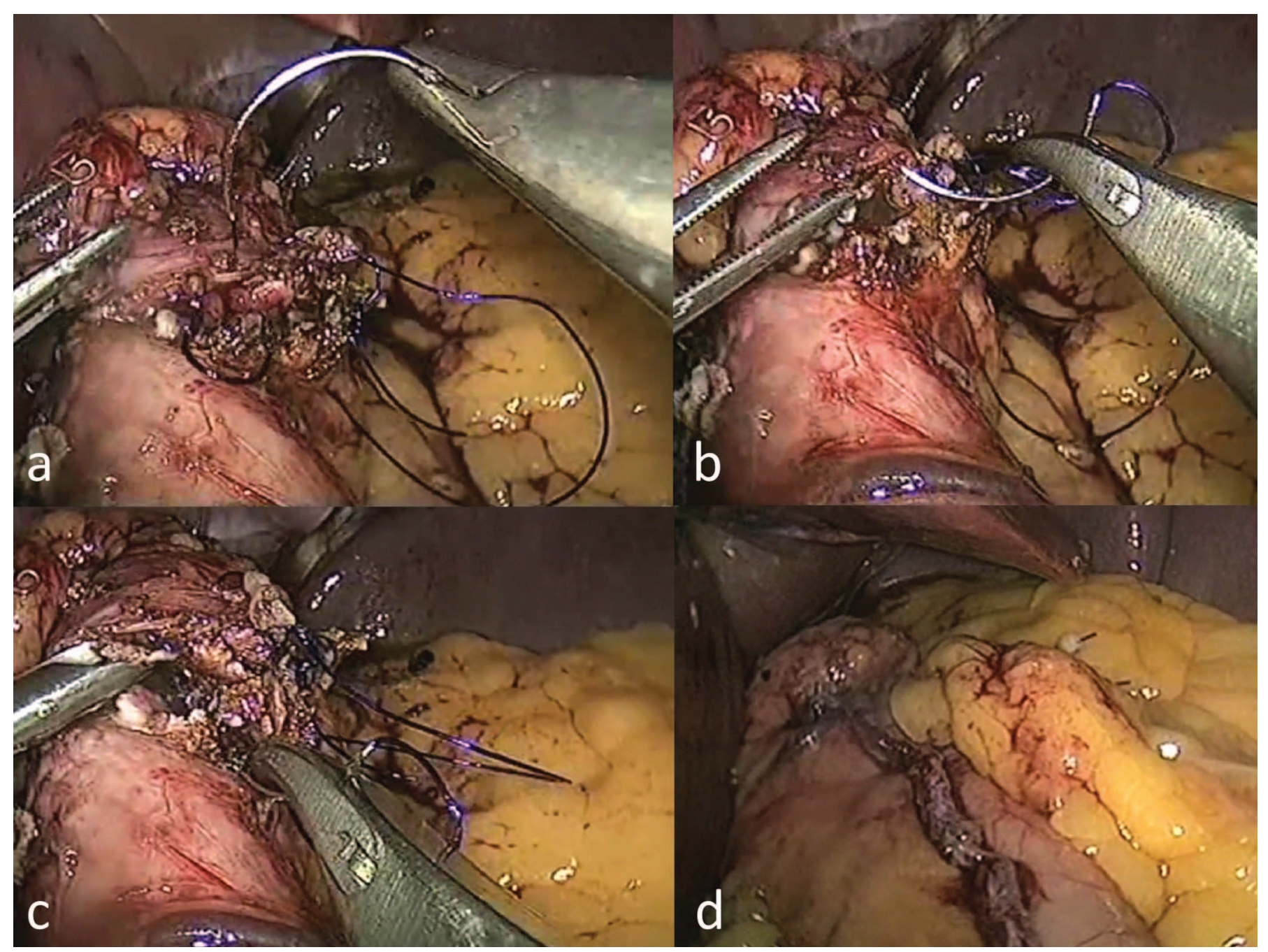

Figure 2. A-C, Transversal closure with a full-thickness running suture of resorbable monofilament material $2 / 0$ to complete the stricturoplasty. D, An omental patch was added over the SP.

the conclusion of the procedure to evaluate the final shape of the sleeve and to make sure there were no leaks. An omental patch was added over the SP (Figure 2D). A drain was left in the abdominal cavity along the SG and remained until discharge from the hospital. A nasogastric (NG) tube was placed per routine for 24 hours. We removed the NG tube after 24 hours once a diatrizoic acid swallow did not show an acute leak. The postoperative course was uneventful, and the patient was discharged on postoperative day 3. Total parenteral nutrition was not required. She was instructed to follow a liquid to semiliquid diet for 2 weeks. At 8-month follow-up, no concern in terms of weight loss, strictures, or stenosis had appeared.

\section{Case 2}

A 29-year-old woman was admitted to our department to undergo an LSG. Her weight was $98 \mathrm{~kg}$ and her height was $164 \mathrm{~cm}$ (body mass index $36 \mathrm{~kg} / \mathrm{m}^{2}$ ). She had an adjustable lap band in place, and it was removed and an LSG performed in one stage at the end of the procedure. A stenosis was detected at the level of the incisura angularis. The intraoperative iatrogenic stenosis was diagnosed by observation and also after air insufflation demonstrated the final shape of the gastric sleeve. A decision was made to perform an SM to address the condition (Figure $\mathbf{3 A}$ and $\mathbf{B}$ ). An air leak test was done before covering the area of the SM with an omental patch (Figure 3C and $\mathbf{D}$ ). Total parenteral 
Intraoperative Prevention of Stenosis for Laparoscopic Sleeve Gastrectomy, Vilallonga R et al.

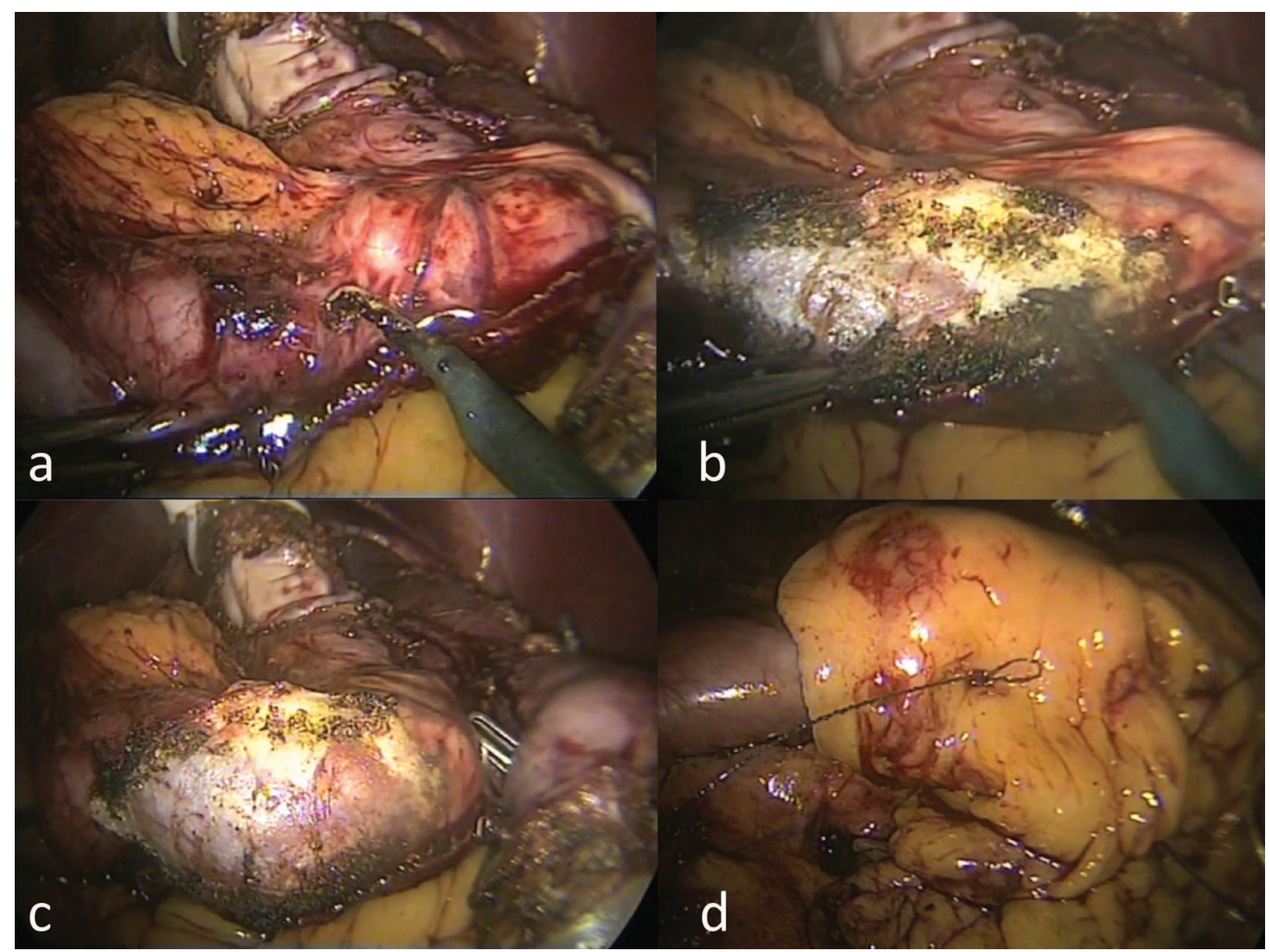

Figure 3. A, A stenosis is detected at the level of the incisura angularis. B, Complete SM to address the stenosis. C and D, An air leak test was done before covering the area of the SM with an omental patch.

nutrition was not required. The patient was discharged on postoperative day 4 after an uneventful recovery. On the midterm follow-up (9 months), no relevant problems had appeared.

Both patients will be followed in the outpatient clinic every 3 months during the first year and every 6 months in the following year (year 2).

\section{DISCUSSION}

Among all of the complications that may occur after an LSG, some such as leak or bleeding can be life threatening. ${ }^{4,5}$ Other complications such as chronic strictures may require revisional surgery because of their incapacitating symptoms. ${ }^{6}$ The incidence of long-term stric- tures is reported to be $\approx 3.5 \% .^{7}$ An avoidable cause of stricture is a stapling error (ie, stapling may be accidentally performed at too sharp an angle, creating too narrow a sleeve). This flaw most often occurs at the level of the incisura angularis, as seen in the second case. ${ }^{3}$ Another cause of strictures is nonsymmetrical traction applied during stapling because it may generate a narrowing of the lumen with a twist of the sleeve. Both aforementioned conditions create a stenosis despite the presence of a $34-\mathrm{Fr}$ bougie as seen in Case 1 (Figure 1A). When the stenosis is recognized, either by simple observation (retraction of the gastric sleeve after removal of the bougie) or by an air insufflations test, different approaches can be considered to address this condition. First, the sleeve may be converted to a gas- 
tric bypass. However, this implies a different surgical philosophy, hence physiologic consequences that are different from the intended ones. Second, intraoperative SM may be performed (Case 2) as has previously been proposed by our group in the setting of a chronic stricture. ${ }^{8}$ Despite the fact that in our experience SM (for chronic sleeve gastrectomy stenosis) had a significant risk of leak (35\%), a better adapted technique including avoiding electrocautery and a systematic omental patch to cover the SM may decrease these mishaps. 9 However, this preliminary experience describes the management of chronic and not acute stenosis during the primary procedure where tissues offer better characteristics. Finally, as shown in Figures 1 and 2, SP can be used intraoperatively. This technique appears to be safe and straightforward. An SP can be performed in case of failed SM with a serosa perforation. Conversion from an SM to an SP can be done. The SP must address the entire stenotic area. Once performed, the incision is closed transversely. We used a full-thickness running suture. As long as the stenosis is not too long, a tailored widening of the sleeve can be obtained. Potential hazards related to the SM technique may not be shared by this technique.

\section{CONCLUSION}

Strictures may be prevented by an accurate and standard technique during LSG. SP and SM can be performed to address intraoperative stenosis while preserving the integrity of the sleeve.

\section{References:}

1. Brethauer SA, Hammel J, Schauer PR. Systematic review of sleeve gastrectomy as a staging and primary bariatric operation. Surg Obes Relat Dis. 2009;5:469-475.

2. Tucker ON, Szomstein S, Rosenthal RJ. Indications for sleeve gastrectomy as a primary procedure for weight loss in the morbidly obese. J Gastrointest Surg. 2008;12:662-667.

3. Rosenthal RA. International Sleeve Gastrectomy Expert Panel Consensus Statement: Best Practice Guidelines Based on Experience of $>12,000$ Cases. Surg Obes Relat Dis. 2012;8:8-19.

4. Aurora AR, Khaitan L, Saber AA. Sleeve gastrectomy and the risk of leak: a systematic analysis of 4,888 patients. Surg Endosc. 2012.;26:1509-1515.

5. Lalor PF, Tucker ON, Szomstein S, Rosenthal RJ. Complications after laparoscopic sleeve gastrectomy. Surg Obes Relat Dis. 2008;4:33-38.

6. Zundel N, Hernandez JD, Galvao Neto M, Campos J. Strictures after laparoscopic sleeve gastrectomy. Surg Laparosc Endosc Percutan Tech. 2010;20:154-158.

7. Parikh A, Alley JB, Peterson RM, et al. Management options for symptomatic stenosis after laparoscopic vertical sleeve gastrectomy in the morbidly obese. Surg Endosc. 2012;26:738-746.

8. Dapri G, Cadière GB, Himpens J. Laparoscopic seromyotomy for long stenosis after sleeve gastrectomy with or without duodenal switch. Obes Surg. 2009;19:495-499.

9. Vilallonga R, Himpens J, Van de Vrande S. Laparoscopic management of persistent strictures after laparoscopic sleeve gastrectomy. Obes Surg. 2013;23(10):1655-1661. 